\title{
THEORY OF A DOUBLE-DiAPHRAGM MEMS UltRASONIC TRANSDUCER
}

\author{
D.W. Greve \\ Department of Electrical and Computer Engineering, Carnegie Mellon University, Pittsburgh, PA, USA \\ I.J. Oppenheim \\ Department of Civil and Environmental Engineering, Carnegie Mellon University, Pittsburgh, PA, USA
}

\begin{abstract}
Conventional capacitive MEMS transducer designs feature a single flexible diaphragm acting as a moving plate and an electrode patterned on the substrate acting as a stationary plate. Such devices generally function adequately as receivers. However, such devices show poor transmission efficiency when the gap dimension is limited by the manufacturing process and when the diaphragm would suffer "pull-in" collapse if deflected electrostatically beyond a critical fraction of the gap dimension. We describe a novel design, one that can be easily fabricated within an existing multi-layer polysilicon process, to achieve more efficient emission. The design features two flexible diaphragms to be actuated by a coordinated series of pulses. We report predictions of the improvement using dimensions characteristic of a multi-user surface-machined MEMS process.
\end{abstract}

\section{INTRODUCTION}

Several groups have investigated MEMS transducers for ultrasonic transmission and reception $[1,2,3,4]$. Much of this work utilizes custom processes that make it possible to achieve high performance, both through optimization of device dimensions and through minimization of parasitics. Recently we explored the use of the multi-user MUMPS process [5] for the fabrication of MEMS ultrasonic transducers $[6,7]$. One problem we encountered is relatively poor transmission efficiency, which is a consequence of the relatively large inter-electrode gaps $(0.75-2 \mu \mathrm{m})$ in the MUMPS process. In this paper we investigate techniques that can theoretically improve the performance of such transducers. While this work was motivated by the limitations and possibilities within the MUMPS process, these techniques may have application elsewhere.

Our design features two flexible diaphragms to be actuated by a coordinated series of pulses. The two flexible diaphragms and the stationary plate form three (upper, middle, and lower) parallel electrodes, and force can be exerted on the middle diaphragm by either the upper or the lower electrodes. A pulse on the upper electrode will reduce the gap between it and the middle diaphragm, and pulses on both upper and lower electrodes will then produce a force on the upper electrode that is larger than that obtained in a conventional device, while simultaneously restraining the middle electrode from excessive movement. In this way, the conversion of electrical to ultrasonic energy is more efficient, while collapse is prevented. We present a theory describing the improved performance of the double-diaphragm in transmission, and we present simulations, using typical dimensions that would be employed with the MUMPS process, comparing the emission performance of the novel design to that of the conventional design.

\section{DOUBLE-DIAPHRAGM STRUCTURES}

Figure 1 shows a plan view and two cross-sections of a structure suitable for fabrication in the MUMPS process. The process has three structural polysilicon layers, two of which can be released to form diaphragms. It is thereby possible to fabricate and release a middle diaphragm in POLY1, suspended between the upper (POLY2) and lower (POLY0) layers. Voltages can be applied independently to the top and bottom electrodes, resulting in both upward and downward forces on the middle diaphragm. We discuss here the use of this internal diaphragm to improve transmitting and receiving efficiency.

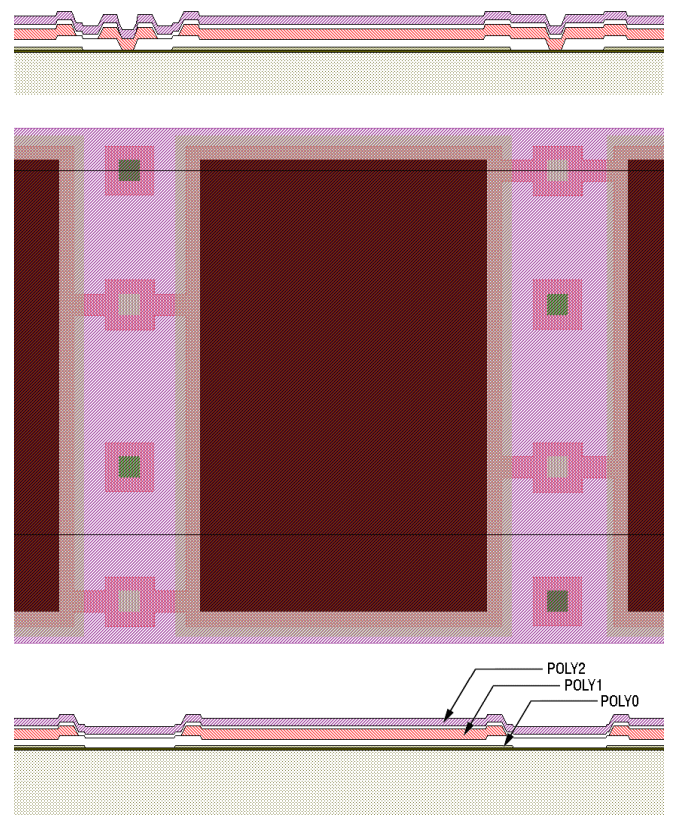

Fig. 1 Plan view and cross sections showing double released diaphragm structure fabricated in the MUMPS process. Etch release holes are omitted for simplicity.

Figure 1 shows a possible mask layout forming a middle diaphragm in the POLY1 layer suspended by short springs. 
For reasons that will become apparent, it will generally be advantageous for the resonant frequency of the middle diaphragm to be low in comparison to the resonant frequency of the upper diaphragm, for air-coupled applications, and to be low in comparison to the forcing frequency, for fluid- or solid-coupled applications. The spring constants suspending the middle diaphragm can be easily controlled, and will certainly be lower than the effective spring constant for a continuous POLY2 (upper) diaphragm supported along its edges. Consequently, it is straightforward to design the middle (internal) diaphragm to have a relatively low resonant frequency. The diaphragms are released by removing sacrificial $\mathrm{SiO}_{2}$ layers through etch release holes, which are omitted in Fig. 1 for simplicity. The thickness of the POLY1 layer is $2.0 \mu \mathrm{m}$, the gap between POLY0 and POLY1 is $1.25 \mu \mathrm{m}$ (when the DIMPLE mask is used), and the gap between POLY1 and POLY2 is $0.75 \mu \mathrm{m}$.

\section{TRANSIENT EXCITATION OF DOUBLE DIAPHRAGM COUPLED TO SOLID OR LIQUID}

We explore the application of coordinated voltage waveforms on the upper and lower plates in order to increase the emitted energy when compared to the case of a conventional single diaphragm device. In principle, it should be possible first to deflect the middle diaphragm toward the upper diaphragm, reducing the gap, and then to apply the forcing voltages to the upper and lower plates. By applying the voltage to the upper plate across a reduced gap, the force on the upper plate and thus the emitted ultrasonic energy will be increased.

In imaging and fault detection applications, it is necessary to emit very short ultrasonic pulses, with Fourier components distributed around a center frequency typically ranging from $1 \mathrm{MHz}$ to $10 \mathrm{MHz}$. As the total pulse duration is very short, it is both desirable and feasible to choose operating conditions so that the middle diaphragm, previously deflected to narrow the gap, does not deflect significantly during the pulses. The deflection must not increase to the point that the middle diaphragm contacts the upper diaphragm, should not decrease and thereby move away from the desired narrowed gap condition, and must not decrease to the point that the middle diaphragm contacts the lower plate. Three factors can be exploited to limit motion of the middle diaphragm: the inertial mass of the diaphragm; opposing force imposed by voltage applied to the lower electrode; and viscous damping. To illustrate the possibility of improved emission in a straightforward way, we first consider balancing the forces on the middle diaphragm.

Figure 2 shows a sketch of the double-diaphragm emitter. Attractive forces can be applied to the middle diaphragm by the voltages $v_{1}(t)$ and $v_{2}(t)$.

We note first that the upper diaphragm is essentially stationary when coupled to a solid or liquid medium. This can be verified by examining the force applied to the upper diaphragm, which is given by

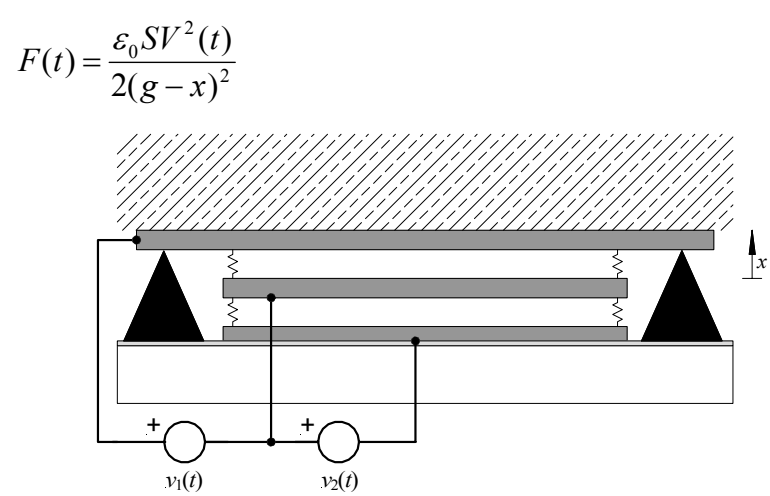

Fig. 2. Sketch of a double-diaphragm emitter.

For a rough estimate of the upper diaphragm deflection, we assume $V(t)=V_{a} \sin (\omega t)$; neglecting constants and the second harmonic we have

$P(t)=\frac{F(t)}{S}=\frac{\varepsilon_{0} V_{a}^{2} \sin (\omega t)}{2(g-x)^{2}}$

Recognizing that the acoustic impedance $Z=P / u$ we have $u(t)=P(t) / Z$ and integrating to get the deflection $x$ yields the result

$x_{\max }=\frac{1}{\omega} \frac{\varepsilon_{0} V_{a}^{2}}{2 Z(g-x)^{2}}$.

Assuming a water medium $\left(Z=1.48 \times 10^{5} \mathrm{gm} / \mathrm{cm}^{2} \mathrm{sec}\right)$, with $\omega=2 \pi \times 5 \mathrm{MHz}, g-x=0.5 \mu \mathrm{m}$, and $V_{a}=100 \mathrm{~V}$, we obtain a maximum deflection of about $0.0076 \mu \mathrm{m}$. We conclude that the upper diaphragm deflection is insignificant in comparison to the gap dimension.

We therefore investigate the motion of the middle diaphragm assuming the upper diaphragm to be fixed. The ultrasonic energy emitted into the fluid of solid medium is calculated from the pressure exerted on the upper diaphragm. For simplicity, in this first analysis we assume that the gaps between upper and lower diaphragms are equal, and that the areas are also equal.

The single-degree-of-freedom equation of motion of the middle diaphragm is

$m \ddot{x}=-k x-b \dot{x}+\frac{\varepsilon_{0} S v_{1}^{2}(t)}{2(g-x)^{2}}-\frac{\varepsilon_{0} S v_{2}^{2}(t)}{2(g+x)^{2}}$

where $S$ is the area of the middle diaphragm, $g$ is the gap, $k$ is the spring constant, $b$ the damping coefficient, and $m$ is the diaphragm mass.

We assume that the applied voltage is limited to $2 V_{a}$, in order to avoid breakdown or for other practical reasons. If the middle diaphragm is deflected toward the upper plate, clearly the force exerted on it by the lower plate will be reduced. Suppose the waveform applied to the upper plate is given by $v_{1}(t)=V_{a}(1+\sin (\omega t))$. Since this voltage is applied across a reduced gap, it is desirable to exert the maximum possible force from the lower plate by choosing $v_{2}(t)=2 V_{a}$. There is a deflection $\Delta$ which results in exactly 
zero average force on the middle diaphragm; this is the solution to the equation

$$
\frac{4 V_{a}^{2}}{(g+\Delta)^{2}}=\frac{V_{a}^{2}}{(g-\Delta)^{2}} \int_{0}^{2 \pi}[1+\sin (\omega t)]^{2} d(\omega t)
$$

or $\Delta=\left(2^{3 / 2}-3^{1 / 2}\right) g /\left(2^{3 / 2}+3^{1 / 2}\right) \approx 0.24 g$. At this gap, for the averaged condition posed above, the force exerted on the upper electrode is increased by about a factor of two, or the ultrasonic power increased by a factor of four, with respect to a single diaphragm with a fixed gap.

This assumes that the middle diaphragm remains stationary at a deflection of $0.24 \mathrm{~g}$, which is not the exact case. Therefore, we use simulation to determine the position of the middle diaphragm as a function of time, and also to determine the force on the upper electrode. There are many variables to explore so we limit these calculations to a few specific cases. We have assumed that the exciting waveform is at a frequency $10 \times$ greater than the resonant frequency of the middle diaphragm.

We show simulations for a middle diaphragm that corresponds to a particular diaphragm that we have fabricated in the MUMPS process and studied experimentally [8]. In this simulation, performed using Mathcad $^{\mathrm{TM}}$, we now use the real MUMPS gap dimensions of 1.25 and $0.75 \mu \mathrm{m}$ as discussed earlier. The single-degree-of-freedom parameters are shown in Table I. The middle diaphragm is given a damping $\mathrm{Q}=3$, which can be adjusted by varying the size and spacing of etch release holes in the middle electrode [9].

Table I. Parameters for single-degree-of-freedom system.

\begin{tabular}{|c|c|}
\hline Parameter & value \\
\hline $\mathrm{m}$ & $0.2 \times 10^{-9} \mathrm{~kg}$ \\
\hline $\mathrm{k}$ & $10^{3} \mathrm{~N} / \mathrm{m}$ \\
\hline $\mathrm{S}$ & $0.45 \times 10^{-6} \mathrm{~m}^{2}$ \\
\hline $\mathrm{Q}$ & 3 \\
\hline $\mathrm{f}_{0}$ & $356 \mathrm{kHz}$ \\
\hline
\end{tabular}

The driving waveform for $v_{l}(t)$ is shown in Fig. 3; this waveform is chosen to be similar to the short pulses typically used for ultrasonic imaging and flaw detection. When a voltage $v_{2}(t)$ is applied to the lower electrode, it is given the same maximum amplitude as $v_{l}(t)$ and shaped by the same triangular envelope.

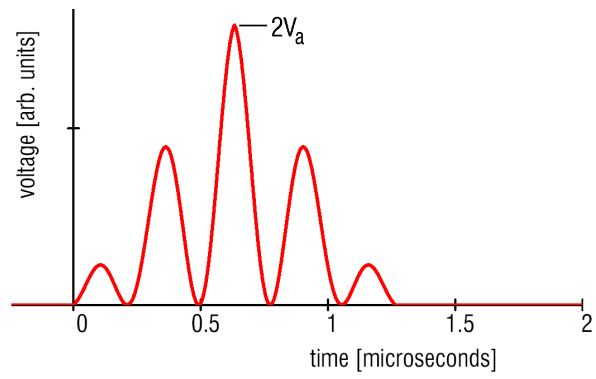

Fig. 3. Waveform $v_{l}(t)$ on the upper electrode.
We consider first the effect of driving the upper electrode with $v_{l}(t)$, with no bias applied to the lower electrode. Our initial condition involves positioning the middle diaphragm at $\mathrm{x}=0.25 \mu \mathrm{m}$ with zero velocity, which may be achieved with an appropriate preparatory pulse. (In this discussion the initial displacement has been taken as one-third of the gap, although other values can equally be investigated.) Figure 4 then shows the subsequent calculated displacement of the middle diaphragm for two cases: a maximum pulse amplitude of $48 \mathrm{~V}$, for which the middle diaphragm is shown to approach but not quite contact the upper electrode, and no voltage pulse, for which the middle diaphragm follows a damped free vibration. The maximum amplitude of $48 \mathrm{~V}$ is considerably larger than the nominal pull-in collapse voltage of the middle diaphragm, which is 17.1 V. With well-chosen pulses applied to the upper electrode, the force on the middle diaphragm causes it to approach, but not strike the upper electrode. In this case the middle electrode is kept from striking the upper electrode by its inertia and by damping.

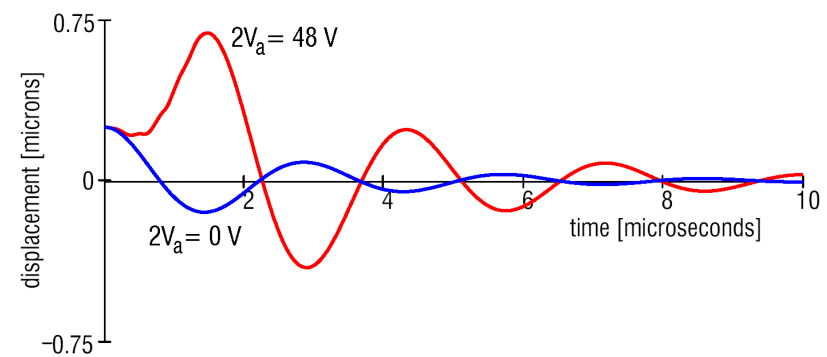

Fig. 4. Simulated position of middle diaphragm as a function of time with initial condition at $x=g / 3$. Trajectories are shown for no applied voltages and with a pulse with $48 \mathrm{~V}$ maximum amplitude applied to the upper electrode only.

We now consider the effect of voltage $v_{2}(t)$ applied to the lower electrode. Figure 5 shows the simulated displacement of the middle diaphragm with and without voltage applied to the lower electrode. With $v_{2}(t)$ applied to the lower electrode, the amplitude of the exciting pulse $v_{l}(t)$ can be increased to $28.2 \mathrm{~V}$, or $56.4 \mathrm{~V}$ max. In the absence of $v_{2}(t)$, there would be a collision between upper and middle electrodes at that same exciting pulse $v_{l}(t)$.

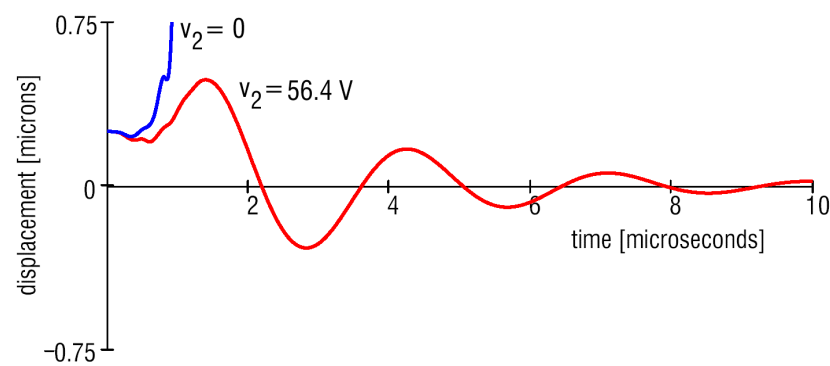

Fig. 5. Simulated position of middle diaphragm as a function of time with initial condition $x=g / 3$. Trajectories are shown for $56.4 \mathrm{~V}$ maximum amplitude applied to the upper electrode, with voltages of zero or $56.4 \mathrm{~V}$ maximum amplitude applied to the lower electrode. 
We now estimate the improvement in emitted ultrasonic energy. Since the velocity $u(t)=P(t) / Z=F(t) S / Z$ we have for the ultrasonic energy

$$
E \sim \int_{\text {pulse }} F^{2}(t) d t .
$$

We have calculated the force on the upper electrode, for the maximum pulse amplitude of $56.4 \mathrm{~V}$, and compared that force to the force calculated across the original gap dimension. We calculate an approximate doubling of the force, leading to an approximate factor of four increase in the emitted ultrasonic energy.

We see that the double-diaphragm approach results in a useful increase in the performance of MUMPS ultrasonic emitters under the assumption of a fixed maximum pulse voltage. There is a practical limit to the improvement that can be obtained even if the pulse voltage is allowed to increase further. For sufficiently large pulse voltages the middle diaphragm experiences unbalanced forces large enough to result in collision with the upper electrode.

\section{RECEIVE PERFORMANCE OF DOUBLE-DIAPHRAGM}

When the upper and middle diaphragms are operated as a capacitive-type detector, for the cases of fluid- and solidcoupled applications the DC bias voltage has the effect of deflecting the middle diaphragm, and for the case of an aircoupled application the DC bias voltage has the effect of deflecting both diaphragms. As a result, the gap is narrowed and improved sensitivity is achieved. The output current is given by the expression [6]

$i=V_{D C} \frac{\varepsilon_{0} S}{(g-x)^{2}} \frac{d x}{d t}$

With voltage applied only between the top and middle diaphragms, pull-in collapse occurs for a displacement of $\mathrm{g} / 3$. Limiting the displacement to $\mathrm{g} / 4$ for safety results in an increase in signal current of approximately a factor of 1.8 . Note that it is easy to engineer the spring supports of the middle diaphragm to obtain the desired deflection at the operating voltage.

\section{CONCLUSIONS}

It is feasible to fabricate a double-diaphragm device within the MUMPS process, and theoretical improvements in the emission and reception performance of a capacitive device have been examined. The improvement results from the ability to deflect the middle diaphragm and thereby narrow the gap between it and the upper plate. For the case of emission, the dynamics of the middle diaphragm must be considered and a coordinated pair of voltages must be applied to the upper and lower plates. However, simulations indicate that practical coordinated voltages can be used, with an approximate $400 \%$ improvement in emitted energy when compared to the conventional case of a capacitive device with a fixed gap.

\section{ACKNOWLEDGEMENTS}

This work is supported by the National Science Foundation under grant CMS-0329880 and by the Commonwealth of Pennsylvania through the Pennsylvania Infrastructure Technology Alliance program.

\section{REFERENCES}

[1] "Surface micromachined ultrasound transducers in CMOS technology," P.-C. Eccardt, K. Niederer, T. Scheiter, and C. Hierold, 1996 IEEE Ultrasonics Symposium, pp. 959-962 (1996).

[2] "Micromachined ultrasonic capacitance transducers for immersion applications," A.G. Bashford, D.W. Schindel, D.A. Hutchins, IEEE Transactions on Ultrasonics, Ferroelectrics, and Frequency Control, vol. 45, pp. 367-375 (1998).

[3] "Characterization of one-dimensional capacitive micromachined ultrasonic immersion transducer arrays," X. Jin, I. Ladabaum, F.L. Degertekin, S. Calmes, and B.T. Khuri-Yakub, IEEE Journal of Microelectromechanical Systems, vol. 8, pp. 100-114 (1999).

[4] "Characterization of one-dimensional capacitive micromachined ultrasonic immersion transducer arrays," X. Jin, O. Oralkan, F.L. Degertekin, and B.T. KhuriYakub, IEEE Transactions on Ultrasonics, Ferroelectrics, and Frequency Control, vol. 48, pp. 750-760 (2001).

[5] MUMPs Design Handbook, D. Koester, R. Mahadevan, B. Hardy, and K. Markus, Cronos Integrated Microsystems, Research Triangle Park, NC, 2001

[6] "MEMS Ultrasonic Transducers for the Testing of Solids," I.J. Oppenheim, A. Jain, and D.W. Greve, IEEE Transactions on Ultrasonics, Ferroelectrics, and Frequency Control, vol. 50, pp, 305-311 (2003).

[7] "MEMS Phased Array Detection in Contact with Solids," D.W. Greve, A. Jain, and I.J. Oppenheim, 2003 IEEE Ultrasonics Symposium, pp. (2002).

[8] "Robust Capacitive MEMS Ultrasonics Transducers for Liquid Immersion," D.W. Greve, J.J. Neumann, I.J. Oppenheim, S.P. Pessiki and D. Ozevin, (to be presented at the 2003 IEEE Ultrasonics Symposium, Honolulu, Hawaii).

[9] "Electrical Characterization of Coupled and Uncoupled MEMS Ultrasonic Transducers," I.J. Oppenheim, A. Jain, and D.W. Greve, IEEE Transactions on Ultrasonics, Ferroelectrics, and Frequency Control, vol. 50, pp, 297-304 (2003). 